\title{
El archivo de la Dirección Federal de Seguridad: una fuente para escribir la historia de la segunda mitad del siglo XX mexicano
}

The Dirección Federal de Seguridad Archive: Sources for Writing the History of the Second Half of the Twentieth Century in Mexico

\author{
Mónica Naymich López Macedonio \\ Escuela Nacional de Estudios Superiores, Unidad Morelia \\ Universidad Nacional Autónoma de México \\ utopistica@gmail.com
}

Recibido: 10 de enero de 2018 - Aprobado: 21 de febrero de 2018

Cómo citar este artículo: Mónica Naymich López Macedonio, "El archivo de la Dirección Federal de Seguridad: una fuente para escribir la historia de la segunda mitad del siglo xx mexicano", en Legajos. Boletín del Archivo General de la Nación, núm. 15 (enero-abril 2018), pp. 71-82.

\section{Resumen}

El archivo de la Dirección Federal de Seguridad (DFS) no es solo el archivo de la Guerra Sucia. Se trata de una fuente imprescindible para reconstruir una historia de México más amplia. Este artículo tiene el propósito de mostrar algunas de las posibilidades de investigación logradas a partir de ese acervo, que nos dejan ver el amplio horizonte y la complejidad de los actores sociales y políticos de la segunda mitad del siglo xx; un periodo que hasta hace poco más de una década parecía inédito para los historiadores en México, porque había sido explorado y tratado, principalmente, por la audacia de aquellos que ejercen el periodismo como profesión.

Palabras clave: DFS, DIPS, Cisen, Guerra Fría, anticomunismo, Estado corporativo mexicano, movimientos estudiantiles, movimientos armados

\section{Abstract}

The Dirección Federal de Seguridad (DFS) archive covers more than the Dirty War. The collection is an crucial source for anyone who wishes to reconstruct broader histories of Mexico. This article highlights the research possibilities that this valuable collection offers. The archive allows us to see the broad horizon and complexity of the social and political actors in Mexico during the second half of the twentieth century - a period overlooked until recently by historians, who left it to researchers bold enough to practice professional journalism.

Keywords: DFS, DIPS, Cisen, Cold War, Anticommunism, Mexican Corporate State, Student Movements, Armed Movements 
La historiografía producida durante el siglo xx ha sido muy generosa con el estudio del periodo novohispano, el siglo xix y con las primeras cuatro décadas del siglo xx mexicano. Pero después de ese gran momento que significó para el país, la Revolución mexicana, con sus instituciones y el gobierno cardenista, fue quedando pendiente por explicar el desenlace de ese movimiento y lo que ha quedado de ella en los albores del siglo xxi. He aquí, una de las enormes posibilidades de investigación que ofrece el archivo de la DFs. Su consulta libre y sin candados, puede significar el advenimiento de novedosas investigaciones y perspectivas de análisis sobre lo que fue el Estado mexicano y sus instituciones, la complejidad de la política, la cultura y la sociedad del México de la segunda mitad del siglo xx. Por supuesto que existen otro tipo de fuentes que nos permitirían escribir historias acerca de ese periodo. Pero el archivo de la DFs ha logrado ser preservado y sería una pena prescindir de él, ya sea por la censura gubernamental o por la autocensura de aquellos que la consideran una fuente "poco confiable" porque reproduce imprecisiones y rumores. Los miles de expedientes que lo componen no están exentos de ese tipo de problemáticas las cuales, sin embargo, no son exclusivas de los informes de la DFs; esta documentación, como todo vestigio del pasado, requiere de una necesaria crítica de fuentes. El archivo de la DFs es un extraordinario acervo que forma parte importante de nuestra memoria nacional, registro de lo que hemos sido, el cual debe ser entendido como parte de nuestro patrimonio histórico cultural. A través de los millones de expedientes, fotografías y videos que lo componen, podemos descifrar claves de la política, la sociedad y la cultura mexicana que reclaman la pluma de su investigador.

El archivo de la DFs ofrece un panorama muy completo del pulso nacional del periodo que va desde 1947 hasta 1985. Los agentes en nómina de la DFs estuvieron distribuidos en todo el territorio nacional y vigilaron tanto a "los amigos como a los enemigos del régimen". Estos agentes en nómina de la DFs colaboraron con ella a través de su infiltración en los más diversos espacios e instituciones de la sociedad mexicana. Existieron también los "agentes honorarios", es decir, aquellos que no estuvieron en nómina de la DFs y que sí lo 
estaban en las nóminas de otras instituciones de las que formaban parte y de las que rindieron informes periódicamente. La distinción entre agentes honorarios y agentes en nómina de la DFs, así como la riqueza de sus informes, fue advertida por el profesor-investigador de El Colegio de México, Sergio Aguayo Quezada, en su libro pionero sobre los servicios secretos mexicanos que fue, además, la primera investigación histórica que se realizó a partir de ese importante acervo documental. ${ }^{1}$ La DFs fue la principal policía política de los gobiernos del Partido Revolucionario Institucional (PRI). Fue creada en 1947 y dejó de operar en 1985 cuando, a decir de Sergio Aguayo Quezada, se hizo pública su degradación y corrupción. En su lugar fue erigida la Dirección General de Investigación y Seguridad Nacional en 1986, que fusionó las labores de la DFs y la Dirección General de Investigaciones Políticas y Sociales (DGIPS) esta última, con tareas de espionaje e investigación y que operaba en forma paralela y coordinada con la DFs.

El 13 de febrero de 1989, la DGIPs se convirtió en el actual Centro de Investigación y Seguridad Nacional (Cisen) institución que, como sus predecesoras, fue creada como dependencia de la Secretaría de Gobernación. Nueve meses más tarde de la fundación del Cisen, se haría tangible el desmoronamiento de la idea de que el mundo sería algún día la patria del comunismo, como lo evidenció la caída del Muro de Berlín, en noviembre de 1989, y lo confirmaría la desintegración de la Unión de Repúblicas Socialistas Soviéticas (URss) en los albores de la década de 1990; ocasos que anunciaron la gestación de una nueva era para México y para el mundo.

Como evidencia de ello, en México los archivos de la DFs y el de la DIPs se convirtieron en los desclasificados del Cisen y quedaron bajo su custodia. Once años más tarde, teniendo como marco político nacional las grandes expectativas que generó en México la alternancia política de 2000, aquellos fondos documentales fueron trasladados y depositados en el Archivo General de la Nación (AGN), que tiene por sede la Ciudad de México. La intervención de Sergio Aguayo Quezada fue crucial en ese proceso, quien al respecto ha compartido lo siguiente:

${ }^{1}$ Aguayo Quezada, La Charola..., 2001. 
A principios del año 2000 terminaba una historia sobre los servicios de inteligencia mexicanos. Solicité por escrito al Centro de Investigación y Seguridad Nacional (Cisen) autorización para consultar los archivos de la DFs que custodiaba. Me lo concedieron porque anticipaban la derrota del Partido Revolucionario Institucional en las elecciones presidenciales de aquel año; abrirme las puertas era una forma de apertura que permitiría certificar, de manera independiente, que el Cisen había acabado con las prácticas más nocivas de la DFs. Estuve trabajando más de un año en ese acervo y constaté su riqueza. ${ }^{2}$

Sergio Aguayo Quezada añadió que, en julio de 2001, el consejero de Seguridad Nacional, Adolfo Aguilar Zinser, arregló un encuentro personal con el entonces presidente de México, Vicente Fox Quezada, a quien le explicó la trascendencia de depositar los fondos de la DFs y de la DGIPS en el AGN. La conversación tuvo un efecto positivo. El 19 de febrero de 2002 el Cisen hizo la entrega oficial al AGN, de 4223 cajas de los fondos documentales de la DFs y de la DGIPs. El archivo de la DFs se depositó en la Galería 1 y el de la DGips se resguardó en la Galería 2 pero, a diferencia de este último, el archivo de la DFs continuó custodiado por personal del Cisen y no por los archivistas del AGN, como sigue ocurriendo en la actualidad. Desde la llegada de estos acervos al AGN, los investigadores se encontraron con el primer obstáculo: no se conocía con exactitud qué información contenían. Los archivistas del Cisen y el área de Referencias del AGN, comunicaron algunas pistas y las condiciones para su consulta. Solo se podría revisar la información de los organismos e instituciones que fueron vigilados; la información sobre las personas "fichadas" por la DFs y que tenían su respectivo expediente, únicamente podían ser consultadas tras la autorización de esas personas o, en caso de que éstas ya no se encontraran con vida,

${ }^{2}$ Hispanic American Historical Review, "Open Forum on Archives and Access: The DFS Controversy", http://hahr-online.com/open-forum-on-archives-and-access-the-dfscontroversy/ [consultado 6 de diciembre de 2017]. 
por los familiares de las mismas. La expresión estar "fichado" por la DFs, se debió a que su archivista y creador, Vicente Capello y Rocha, sistematizó la enorme información que generaba la DFs en pequeñas fichas "tipo bibliográficas" que sirven de guía para ubicar los contenidos de este acervo. Las fichas de la DFs están mecanografiadas y contienen el nombre e información de la persona u organización que fueron vigiladas. En ellas se mecanografiaron además, el número de la caja, del expediente y un resumen del contenido de los informes periódicos que se generaron a cerca de personas y organizaciones. Las fichas se resguardaron en un archivero de gavetas de metal color gris, en las que estaban organizadas alfabéticamente y por manojos amarrados por ligas de hule, de acuerdo con el criterio antes descrito. Sin saber exactamente qué contenía el acervo y guiados por la intuición y la suerte, los investigadores entregaban una lista con los nombres de las organizaciones y de las personas de las que esperaban encontrar información. La atmósfera que se respiró en la Galería 2, donde fueron depositados los fondos documentales de la DGIPS, permitió a Daniela Spenser llevar a cabo un proyecto de investigación del que resultó Guerra Fría y Guerrilla en México: guía de acceso al Archivo de la Dirección General de Investigaciones Políticas y Sociales, que se editó en 2003. ${ }^{3}$ Apoyado en el archivo de la Dgips, Carlos Inclán Fuentes ha documentado una interesante historia sobre el espionaje y las políticas de control que llevó a cabo el Estado mexicano hacia los ciudadanos alemanes durante la Segunda Guerra Mundial. ${ }^{4}$

Con grandes esperanzas, un gran número de investigadores acudieron a la Galería 1, con la intención de constatar su riqueza por cuenta propia. Para muchos, el archivo de la DFs sería la fuente sobre la que se documentarían las atrocidades cometidas por los gobiernos priistas en el marco de la Guerra Fría, que a su vez legitimó la Guerra Sucia. En este tenor descubrimos los importantes trabajos de Renata Keller (2017). ${ }^{5}$ Las exploraciones en ese archivo trajeron como

\footnotetext{
${ }^{3}$ Spenser (Coord.), Guerra Fría y Guerrilla en México..., 2003.

${ }^{4}$ Inclán Fuentes, Perote y los nazis, 2013.

${ }^{5}$ Keller, "Spies, Assassins, and Statesmen in Mexico's Cold War”, 2017.
} 
resultado investigaciones que documentaron, por ejemplo, historias sobre las guerrillas mexicanas, su desmantelamiento y exterminio por parte del Estado mexicano (2011-2016). ${ }^{6}$ A partir del archivo de la DFs también se documentaron historias sobre el movimiento estudiantil de 1968, enfatizando su lugar en el marco de la Guerra Fría. ${ }^{7}$ Novedosas perspectivas de investigación se han documentado sobre este acervo, entre las que destacan los trabajos de Andrew Paxman $(2017) ;^{8}$ el de Renata Keller sobre el triángulo Cuba, México, Estados Unidos en el marco de la Guerra Fría (2015). ${ }^{9}$

Las inmersiones en ese extraordinario acervo permitieron, además, reconstruir la historia del movimiento anticomunista mexicano, que estuvo constituido por elementos que no formaron parte, al menos formalmente, de las estructuras gubernamentales. ${ }^{10}$ Las investigaciones logradas a partir del acervo de la DFs, permitieron explicar que ese movimiento se constituyó a partir de un sector de la sociedad que había combatido y resistido, al proyecto de nación propuesto por los gobiernos que emergieron de la Revolución Mexicana. Esos sectores mexicanos, que se habían erigido en contra de la Revolución, lograron resistir creando sus propios aparatos de reproducción ideológica, los cuales reivindicaron valores contrarios a los propuestos por las instituciones revolucionarias, exaltaron el valor de la libre empresa, del individualismo emprendedor y los valores de la Iglesia católica tridentina. ${ }^{11}$ Además de hacer del anticomunismo una profesión, los anticomunistas mexicanos tejieron redes en el extranjero y sumaron esfuerzos con organizaciones anticomunistas homólogas que, amparadas en el marco ideológico de la Guerra Fría, se erigieron en los países del continente

\footnotetext{
${ }^{6}$ Gamiño Muñoz y Toledo González, "Origen de la Liga Comunista 23 de Septiembre", 2011; Gamiño Muñoz "La Liga Comunista 23 de Septiembre. Cuatro décadas a debate", 2016.

${ }^{7}$ Collado, "Guerra Fría, movimiento estudiantil de 1968”, 2017.

${ }^{8}$ Paxman, Jenkins of México..., 2017.

${ }^{9}$ Keller, Mexico's Cold War, 2015.

${ }^{10}$ López Macedonio, "Una visita desesperada. La Liga Mundial...”, 2006.

${ }^{11}$ López Macedonio, "La ofensiva anticomunista de los Tecos...”, 2010.
} 
americano, africano, europeo y asiático, para combatir al comunismo. ${ }^{12}$ El estudio del diverso y complejo movimiento anticomunista internacional, es un tema de suma relevancia en la actualidad, porque nos arroja pistas para comenzar a pensar, cómo fue que llegamos a construir un mundo tan distinto al que soñaron los comunistas y cómo se ha hecho realidad el mundo que proyectaron los anticomunistas: el mundo de la empresa privada, el del achicamiento del Estado en la vida económica y social, el de la idea de que solo por medio de la creación de más policías se puede combatir la violencia, entre otros puntos actualmente presentes en algunas de las agendas gubernamentales de las repúblicas democráticas del mundo, incluida la de México.

Pero la riqueza del acervo de la DFs ofrece esa y muchas otras posibilidades de investigación. A partir de él, se puede documentar, por ejemplo, no solo la tolerancia que el Estado mexicano tuvo hacia ciertos opositores - los anticomunistas por ejemplo- durante la segunda mitad del siglo xx. También se pueden hacer visibles los patrones de violencia y de cooptación que el Estado mexicano llevó a cabo en contra de aquellos que, tras haber militado en organizaciones reconocidas por él, se movieron a la disidencia guerrillera o de partido, lo cual nos aporta nuevas perspectivas de análisis sobre esos actores. ${ }^{13}$ Es decir, el archivo de la DFs nos permite conocer cómo funcionó el Estado mexicano antes de la crisis guerrillera y de la Guerra Sucia. A partir de sus miles de expedientes es posible observar el funcionamiento del Estado corporativo mexicano del siglo xx, que existió a partir de las relaciones o pactos políticos laborales que fomentó y construyó con diversos sectores sociales estratégicos, y sobre los que dio soporte a su legitimidad durante varias décadas del siglo xx. Además de los obreros, los campesinos, el sector popular y el magisterio, uno de esos sectores sociales estratégicos, fue el de los estudiantes. Con base en el archivo de la DFs se pueden, por ejemplo, escribir historias de los movimientos

${ }^{12}$ López Macedonio, "Historia de una solidaridad Asiático-Latinoamericana", 2010; López Macedonio, "Historia de una colaboración anticomunista transnacional...", 2010. ${ }^{13}$ López Macedonio, "La construcción del corporativismo estudiantil”, 2012. 
y organizaciones estudiantiles que antecedieron a los álgidos años de 1968 y los de la Guerra Sucia. Las organizaciones estudiantiles precedentes, así como sus movilizaciones, han sido poco exploradas debido, entre otras cosas, al protagonismo y a la trascendencia que adquirió el movimiento estudiantil de 1968. Sin embargo, bien vale la pena comenzar a explorar lo que fue el movimiento estudiantil que le antecedió. En ese precedente existe una línea de investigación que nos arroja importantes luces, por ejemplo, sobre cómo el Estado mexicano construyó su relación con el sector estudiantil y cómo funcionó esa compleja relación política, en el transcurso de la segunda mitad del siglo xx, hasta su ruptura a finales de la década de los sesenta. Antecedentes que nos permiten sostener que los actores que militaron en los movimientos guerrilleros de los años sesenta y setenta del siglo xx, fueron disidentes de la estructura corporativa que he referido y de la que, antes de radicalizarse, habían formado parte. ${ }^{14}$ Los expedientes de -la DFs también nos permiten situar a las organizaciones estudiantiles mexicanas en el marco del movimiento estudiantil internacional, que floreció con peculiar entusiasmo durante los años de la Guerra Fría.

Estas investigaciones son solo la punta del iceberg. A través de ese peculiar acervo se puede advertir y comprender cómo funcionaron las complejas relaciones corporativas que el Estado mexicano cultivó con diversos sectores de la sociedad mexicana, que garantizaron la hegemonía política del PRI durante varias décadas del siglo xx; una estructura corporativa que, por cierto, había sido fraguada en el gobierno cardenista, heredada a los gobiernos priistas, en la que también participaron estudiantes normalistas y universitarios y con la que los políticos priistas gobernaron este país durante el siglo xx; una perspectiva que sugiere revisar la historia de los movimientos armados mexicanos de la década de los sesenta y setenta. ${ }^{15}$ En los archivos de la DFs se puede rastrear la trayectoria de esos actores, antes de su ingreso a la clandestinidad guerrillera, es decir, antes de que se pusiera en práctica la llamada

${ }^{14}$ López Macedonio, "La construcción del corporativismo estudiantil...", 2012.

${ }^{15}$ López Macedonio, "Historia de una relación Institucional”, 2016. 
Guerra Sucia. Esta es solo una de las inmensas posibilidades de investigación que ofrece el archivo de la DFs el cual, como hemos descrito en este texto, no solo es el archivo de la Guerra Sucia.

El archivo de la DFs en México es uno de los acervos que se ha puesto a la consulta pública con una doble función. Es decir, para documentar investigaciones desde la academia y para documentar investigaciones desde la justicia. Al respecto, es importante, al menos enunciar en este espacio que, a la par de la apertura pública de los desclasificados del Cisen en 2002, se creó la Fiscalía Especial para Movimientos Sociales y Políticos del Pasado (Femospp). Este organismo fue creado para documentar los asesinatos y violaciones a los derechos humanos cometidos por servidores públicos de los gobiernos mexicanos de los años sesenta, setenta y ochenta del siglo xx y para, derivado de ese proceso, "fincar responsabilidades jurídicas a los presuntos responsables de dichas conductas delictivas". ${ }^{16}$ Sin embargo, la Femospp fue suprimida en 2007, en el gobierno de Felipe Calderón Hinojosa (2006-2012); el gobierno mexicano que le declaró la guerra al narcotráfico. Con la desaparición de la Femospp se evidenció la pérdida de la voluntad gubernamental para con las familias de los desaparecidos políticos de aquellos años del siglo xx.

Cuando el Partido Revolucionario Institucional regresó al poder en 2012, el acceso a la consulta del archivo de la DFs se fue convirtiendo en una misión más que difícil. Desde 2015, las fichas que servían de guía a los investigadores, fueron retiradas de la consulta abierta y ahora solo es posible tener acceso a los documentos a través de las versiones públicas, es decir, copias de los documentos originales en los que han sido eliminados datos clasificados como confidenciales. Tras el terremoto de septiembre de 2017, el archivo de la DFs fue movido a la galería 4, es decir, sigue depositado en el AGN pero continua custodiado y ahora con más recelo por el personal del Cisen, lo cual representa más que un freno para la investigación histórica de este país.

${ }^{16}$ Femospp, Informe histórico presentado a la sociedad mexicana..., 2008. 


\section{Fuentes}

\section{Bibliográficas}

Aguayo Quezada, Sergio, La Charola. Una historia de los servicios de inteligencia en México, México, Grijalbo, 2001.

Collado, María del Carmen, "Guerra Fría, movimiento estudiantil de 1968 y el gobierno de Gustavo Díaz Ordaz. La mirada de las agencias de seguridad de Estados Unidos”, en Secuencia, núm. 98, mayo-agosto de 2017.

Gamiño Muñoz, Rodolfo, "La Liga Comunista 23 de Septiembre. Cuatro décadas a debate", en De raíz diversa, vol. 3, núm. 5, enerojunio, 2016.

, y Toledo González, Mónica Patricia, "Origen de la Liga Comunista 23 de Septiembre”, en Espiral, vol. 18, núm. 52, Guadalajara, septiembre-diciembre de 2011.

Femospp, Informe Histórico presentado a la sociedad mexicana: Fiscalia especial Femospp. Serie: México: Genocidio y delitos de lesa humanidad. Documentos fundamentales 1968-2008, t. Ix. México, Comité 68, 2008, 890 pp.

Inclán Fuentes, Carlos, Perote y los nazis. Las políticas de control y vigilancia del Estado mexicano a los ciudadanos alemanes durante la Segunda Guerra Mundial (1939-1946), México, unam-Gobierno del estado de Veracruz, 2013.

Keller, Renata, "Spies, Assassins, and Statesmen in Mexico's Cold War", en European Review of Latin American and Caribbean Studies, núm. 3, January-June, 2017.

, Mexico's Cold War. Cuba, the United States, and the Legacy of the Mexican Revolution, Cambridge University Press, 2015. 
López Macedonio, Mónica Naymich, "Una visita desesperada. La Liga Mundial Anticomunista en México y su impacto en Latinoamérica. Notas para reconstruir la historia del movimiento civil anticomunista mexicano", en Fournal of Iberian and Latin American Studies, JILAS, 12:2, December 2006.

, "La ofensiva anticomunista de los Tecos y sus vínculos asiáticos a través de los servicios de inteligencia mexicanos", en Verónica Oikión Solano y Miguel Ángel Urrego Ardila (eds.), Violencia y sociedad. Un hito en la historia de las izquierdas en América Latina, Morelia, México, El Colegio de Michoacán-Instituto de Investigaciones Históricas de la Universidad Michoacana de San Nicolás de Hidalgo, 2010.

, "Historia de una solidaridad Asiático-Latinoamericana", en Lucia Chen y Alberto Saladino, La Nueva Nao: De Formosa a América Latina. Reflexiones en torno al Bicentenario de las Independencias Latinoamericanas, Taipei, Taiwan, Universidad de Tamkang, 2010.

"Historia de una colaboración anticomunista transnacional: Los Tecos de la Universidad Autónoma de Guadalajara y el gobierno de Chiang-Kai-Shek a principios de los años setenta", en Contemporánea. Historia y problemas del siglo xx. Dossier: Enfoques transnacionales de la Guerra Fría en América Latina, Montevideo, Uruguay, Unidad de Comunicación de la Universidad de la República, vol. 1, año $1,2010$.

, "La construcción del corporativismo estudiantil normalista rural durante el gobierno cardenista: el caso de la FECSM", en Memorias del XIII Encuentro Internacional de Historia de la Educación, Zacatecas, México, Agosto de 2012, Memoria electrónica: IsbN 978-607-9087-135.

, "Historia de una relación Institucional. Los estudiantes normalistas rurales organizados en la Federación de Estudiantes 
Campesinos Socialistas de México y el Estado mexicano del siglo xx (1935-1969)", tesis doctoral, Centro de Estudios Históricos, El Colegio de México, diciembre 2016.

Paxman, Andrew, Jenkins of México: How a Southern Farm Boy Became a Mexican Magnate, Nueva York, Oxford University Press, 2017.

Spenser, Daniela (coord.), Guerra Fría y Guerrilla en México: guía de acceso al Archivo de la Dirección General de Investigaciones Políticas y Sociales, México, Centro de Investigaciones y Estudios Superiores en Antropología Social (ciesas), disco compacto, 2003.

\section{Electrónicas}

Hispanic American Historical Review, "Open Forum on Archives and Access: The DFs Controversy", http://hahr-online.com/openforum-on-archives-and-access-the-dfs-controversy/ [consultado 6 de diciembre de 2017]. 